\title{
EUVE Spectra of Coronae and Flares
}

\author{
CAROLE JORDAN
}

Department of Physics (Theoretical Physics), University of Oxford, 1 Keble Road, Oxford, OX1 3NP, UK

Following a summary of early solar EUV spectroscopy the spectra of some late-type stars obtained with the Extreme Ultraviolet Explorer (EUVE) are briefly surveyed. Some transitions which are not included in current emissivity codes but could lead to numerous weak lines, and an apparent continuum in the $E U V E$ short wavelength region, are discussed. The importance of the geometry adopted when interpreting the emission measure distribution is stressed, since radial factors can lead to an apparent emission measure distribution gradient that is steeper than the value of $3 / 2$ expected in plane parallel geometry.

\section{Introduction}

The launch of the Extreme Ultraviolet Explorer (EUVE) began a new era in the study of stellar coronae and stellar flares. The detailed spectra of stars that are being obtained are essential in understanding the physics of stellar coronae and flares, and will aid the interpretation of data with low spectral resolution.

Our present understanding of stellar EUV spectra builds on over 30 years of research in solar spectroscopy, concerning line identifications, plasma diagnostic techniques and methods of modelling from emission line fluxes (see, e.g., Feldman, Doschek, \& Seely 1988; Mason \& Monsignori Fossi 1995; and Jordan \& Brown 1981; respectively).

\section{Early Solar EUV Spectroscopy}

Tousey (1967) reviewed early observations of the solar spectrum in the wavelength range $170 \AA$ to $370 \AA$, which date from 1960 . The group of strong lines between $170 \AA$ and $220 \AA$ were shown to be transitions of the type $3 p^{n}-3 p^{n-1} 3 d$ in Fe VIII to Fe XIV (Gabriel, Fawcett \& Jordan 1966; see also Fawcett 1974, 1981). Flare spectra obtained from instruments on the Skylab Apollo Telescope Mount (ATM) allowed Sandlin et al. (1976) to identify lines of highly ionized iron and of less abundant elements, in the $E U V E$ medium wavelength (MW) range. A more complete list was given by Dere (1978). The lines of Fe XVII $\left(2 p^{5} 3 s-2 p^{5} 3 p\right)$ are of particular interest since this ion is not represented by strong lines in the EUVE short wavelength (SW) region.

Transitions of the type $3 s^{2} 3 p^{n}-3 s 3 p^{n+1}$ in iron lie in both the EUVE MW and long wavelength (LW) ranges. Early spectra contained many second order lines, as do EUVE spectra when the coronal temperature is about $1-2 \times 10^{6} \mathrm{~K}$. The identifications are discussed by Bromage, Cowan \& Fawcett (1978) and in references therein.

In the EUVE SW range the solar spectra analyzed by Malinovsky \& Heroux (1973) still provide a useful comparison for stars which have coronal temperatures around $1-2 \times$ $10^{6} \mathrm{~K}$. In addition to lines of $\mathrm{Ne}, \mathrm{Mg}$ and $\mathrm{Si}$, this region contains many lines originating from transitions in Fe IX to Fe XII of the type $3 p^{n}-3 p^{n-1} 4 s, 4 d$ (e.g., Fawcett et al. 1972), and the $3 p^{n}-3 p^{n-1} 3 d$ transitions in Ni IX - Ni XIV. The first solar flare spectrum was obtained from OSO-5 by Kastner, Neupert, \& Swartz (1974), but see Fawcett \& Cowan (1975) for some revised identifications. The flare spectra are dominated by transitions of the type $2 s^{2} 2 p^{n}-2 s 2 p^{n+1}$ in Fe XVIII to Fe XXIII and provide a useful reference for sources where the temperature is around $6 \times 10^{6} \mathrm{~K}$ to $2 \times 10^{7} \mathrm{~K}$. 


\section{Survey of EUVE Spectra of Coronae and Flares}

\subsection{Procyon: $\alpha$ CMi, F5 IV $-V$}

The spectrum of Procyon is very similar to that of the Sun. The strong group of lines of Fe IX to Fe XI around $171 \AA$ to $180 \AA$ was observed previously at lower resolution from EXOSAT (Schrijver 1985). Observations from the Einstein Observatory were analyzed by Schmitt et al. (1985) and Jordan et al. (1986). The emission measure distribution found by Drake, Laming \& Widing (1995) from the EUVE spectra is consistent with the earlier work, giving a maximum emission measure at about $1.6 \times 10^{6} \mathrm{~K}$. Although the identifications of the stronger lines of iron are secure at the resolution of EUVE lines of elements such as magnesium, silicon and sulphur usually occur in blends. Drake et al. (1995) have deconvolved such blends through spectral modelling. Longer exposures are required to confirm the existence of the weakest lines. Since these provide at least upper limits to line fluxes the conclusion by Drake et al. (1995), that the spectra are best fitted with solar photospheric abundances is unlikely to change. Lines of oxygen and neon are particularly important since they provide the link to the region observed with the International Ultraviolet Explorer (IUE), while silicon is the only element with observable lines formed below and above $2 \times 10^{5} \mathrm{~K}$.

\section{2. $\xi B 00 A(+B) ; G 8 V(+K 4 V)$}

Since observations with IUE show that $\xi$ Boo A dominates the total flux from the system in the UV region, we assume here that the EUVE flux arises mainly from $\xi$ Boo $\mathrm{A}$.

Figure 1 shows segments of the spectra obtained in our Guest Investigator observations. The SW spectrum shows the stronger of the Fe XVIII lines, and probably lines of Fe XIX and Fe XX. A line near $98 \AA$ may not be entirely due to Ne VIII. The MW spectrum shows strong lines of Fe XV and Fe XVI (the latter appear also in the LW spectrum), but only very weak emission in the region of the Fe IX to $\mathrm{Fe} X I$ lines. The group of $\mathrm{Fe}$ XIII lines appears around $202 \AA$, and lines Fe XIV at $211 \AA, 219+220 \AA$ and $264 \AA$ may be detected. Longer exposures are required to confirm the existence of weak features.

\subsection{Capella (G1 III + G8 III) and AU Mic (dM0e)}

These two stars are discussed together since it is useful to make direct comparisons of the calibration spectra. The SW spectra of these stars all show lines of Fe XVIII to Fe XXIII. Fe XIV appears at $192 \AA$, although Ca XVII may also be present. Fe XV is observed in the MW spectrum and Fe XVI in the MW and LW spectra. The line identifications in Capella have been discussed by Dupree et al. (1993). The higher temperature of AU Mic compared to Capella is apparent through the presence of lines of Fe XXII at $114.41 \AA$ and Fe XXI at $102.22 \AA$, which are absent or very weak in Capella. In AU Mic the ratio of the Fe XXII lines to those of Fe XX suggest that the blend at $132.8 \AA$ is dominated by Fe XXIII, rather than Fe XX. Conversely, the line of Fe XIX at $101.55 \AA$ is significantly weaker in AU Mic than Capella. If one examines the precise positions of features near the Fe XXI lines at $97.88 \AA$ and $102.22 \AA$ (whose flux ratio is not sensitive to the electron density), in AU Mic and Capella, it appears that the contribution of Fe XXI to the feature at $98 \AA$ has been overestimated by Dupree et al. (1993). They give $\mathrm{F}(97.88) / \mathrm{F}(102.22)=$ 1.7, whereas Brickhouse et al. (1995) calculate a ratio of 0.44 , closer to the ratio observed in AU Mic. Monsignori Fossi \& Landini (1994) have discussed the spectra of AU Mic.

Regarding electron densities, the ratio of the fluxes of either of the Fe XXI lines at $97.88 \AA$ or $102.22 \AA$ to that of Fe XXI at $128.73 \AA$ is sensitive to $N_{e}$ (Mason et al. 1979). The clear change in the ratios between Capella and AU Mic shows that in AU Mic, at least, the ratio is in the density sensitive regime. Dupree et al. (1993) find a ratio of 

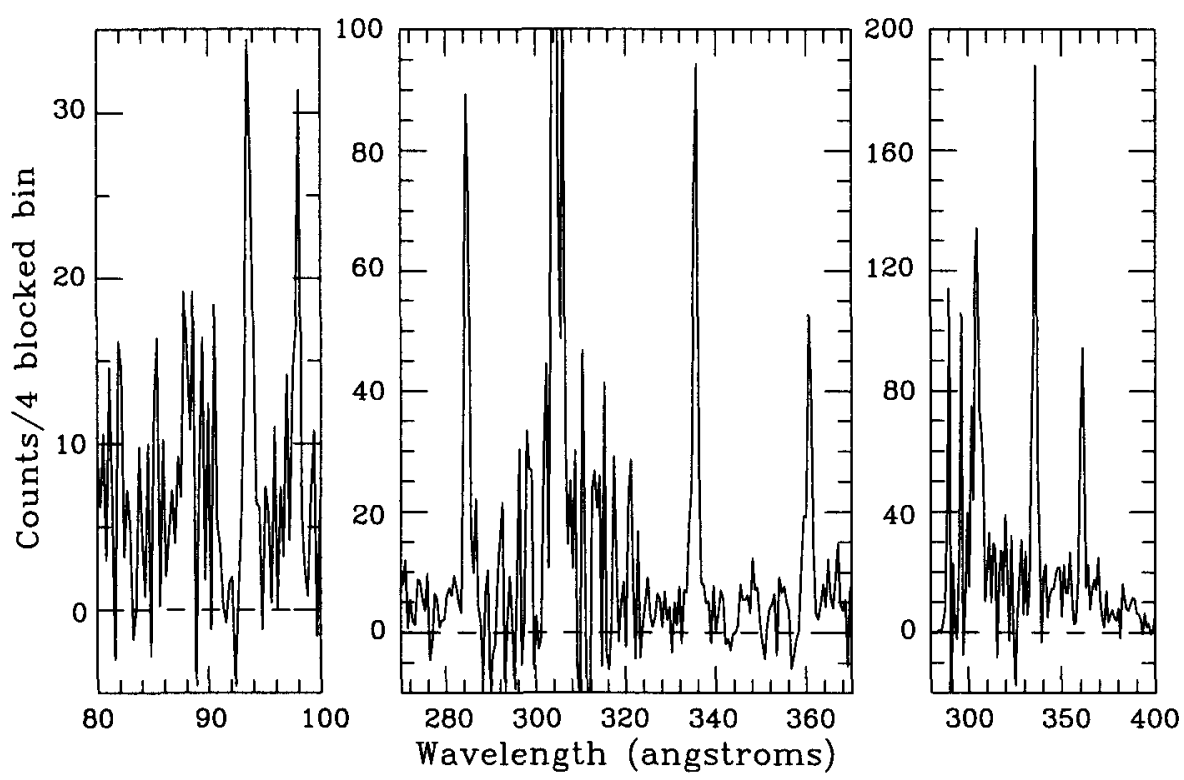

FIGURE 1. EUVE spectra of $\xi$ Boo A, showing lines of Fe XVIII (93.9 $\AA)$, Fe XV $(284 \AA)$ and Fe XVI (335 $\AA$ and $360 \AA)$.

$\mathrm{F}(128.73) / \mathrm{F}(102.22)=4.76$, which is close to the low density limit value of 4.6 from the calculations by Brickhouse et al. (1995). The ratio of these lines in AU Mic leads to a density of about $2-4 \times 10^{12} \mathrm{~cm}^{-3}$ (see also Monsignori Fossi \& Landini 1994). A higher density in AU Mic is also suggested by a doublet transition in Fe XX at $110.63 \AA$, which is absent in Capella. The line of Fe XXI at $146.65 \AA$, whose relative flux increases with $N_{e}$, may just be detectable in $\mathrm{AU}$ Mic, but a longer exposure is required to confirm its presence in Capella. The density in Capella seems to be $\leq 10^{10} \mathrm{~cm}^{-3}$, lower than proposed by Dupree et al. (1993).

\section{Sources of Weak Lines}

It has been proposed that the continuum observed in the EUVE SW region below 150 $\AA$, and the line to continuum ratio, requires an abundance of iron that is lower than in the solar photosphere (e.g., Stern et al. 1995). The present emissivity codes include the stronger lines, but many weaker transitions that could contribute to the continuum are absent. The transitions discussed below need to be further investigated.

\subsection{Weak Lines Formed at $T_{e} \simeq 1-3 \times 10^{6} \mathrm{~K}$}

Although transitions of the type $3 p^{n}-3 p^{n-1} 4 s, 4 d$ in the ions Fe IX to Fe XIV were identified in early solar spectra some lines of moderate strength remained unidentified. Jordan (1968) suggested that these could be $3 p^{n-1} 3 d-3 p^{n-1} 4 p, 4 f$ transitions, excited via the non-dipole transitions $3 p^{n}-3 p^{n-1} 4 p, 4 f$ which can have collision strengths larger than or comparable to dipole transitions. (See reviews of atomic data in Lang 1994). In 
Fe IX to Fe XIV the $3 d-4 p, 4 f$ transitions lie in groups between $117 \AA$ to $73 \AA$, and $170 \AA$ to $91 \AA$, respectively, and some do correspond to lines in the solar spectrum, but not all of the $4 \mathrm{p}$ and $4 \mathrm{f}$ levels are known. When $T_{\text {coron } a} \simeq 1-3 \times 10^{6} \mathrm{~K}$ these lines will contribute to the apparent continuum in the EUVE SW spectra. Mewe, Gronenschild $\&$ van den Oord (1985) do include some of the $3 p^{n}-3 p^{n-1} 4 s, 4 d$ transitions and a few $3 d-4 f$ transitions in Fe XIV to Fe XVI, but the majority of the possible transitions are absent from the current emissivity codes. Much of the atomic data required to predict individual line fluxes has not yet been calculated.

\subsection{Weak Lines Formed at $5 \times 10^{6}$ to $2 \times 10^{7} \mathrm{~K}$}

At these temperatures the strong lines observed in the EUVE SW spectra are of the type $2 s^{2} 2 p^{n}-2 s 2 p^{n+1}$ in Fe XVIII to Fe XXIII. The $2 p^{n}-2 p^{n-1} 3 s, 3 d$ transitions in Fe XVII to Fe XXII occur between $11.8 \AA$ and $17.1 \AA$. Mewe et al. (1985) do include the above transitions, but only some of the non-dipole $2 p^{n}-2 p^{n-1} 3 p$ transitions.

Transitions of the type $2 s^{2} 2 p^{n}-2 s 2 p^{n} 3 s, 3 p$ and $3 \mathrm{~d}$ can also occur, with alternative decay routes in transitions of the type $2 s^{2} 2 p^{n-1} 3 l-2 s 2 p^{n} 3 l$. These $2 s-2 p$ transitions in the presence of a spectator $3 l$ electron lie in broadly the same wavelength region as the strong $2 s^{2} 2 p^{n}-2 s 2 p^{n+1}$ transitions, as will all transitions with a spectator $n l$ $(n \geq 4)$ electron. Apart from Fe XVII, for which Loulergue \& Nussbaumer (1975) made a detailed theoretical study, the energy levels involved and emissivities are mostly unknown. Together, the above transitions in several stages of ionization could appear as a weak continuum in the EUVE SW region.

Solar flare spectra do show transitions with $\Delta n=2$, between about $8 \AA$ and $13 \AA$, e.g., $2 p^{n}-2 p^{n-1} 4 s, 4 d$, but many were identified only recently (Fawcett et al. 1987), and are not systematically included in the emissivity codes. Drake et al. (1994) find that the "MEKA" code (Mewe et al. 1985; Kaastra 1992) underestimates the flux observed in the $A S C A$ spectrum of $\beta$ Cet (K0 III) around $9.5 \AA$, and they increase the abundance of magnesium to achieve a fit. However, the iron lines with $\Delta n=2$ lie around the $\mathrm{Mg} \mathrm{XI}$ (He I-like) lines, and could provide the additional flux without increasing the abundance of magnesium. Decays of the type $3 l-4(l \pm 1)$ are known only in Fe XVII, but in Fe XVII to Fe XXII will lie between about $35 \AA$ to $75 \AA$.

In view of the large number of weak transitions not yet included in the emissivity codes it seems premature to attribute discrepancies between the observations and the predictions of the codes to non-photospheric abundances.

\section{Emission Measures and Their Interpretation}

\subsection{Apparent and True Emission Measures}

The volume emission measure derived from an X-ray flux is usually defined through

$$
F_{\oplus}=\int \epsilon_{\lambda} N_{e}^{2} d V /\left(4 \pi d^{2}\right)
$$

where $\mathrm{d}$ is the distance to the star and $\epsilon_{\lambda}$ is the line emissivity. This expression assumes that all the photons escape from the emitting region without interception by the star and gives an apparent volume emission measure

$$
E m(V)_{a p p}=4 \pi d^{2} F_{\oplus} / \epsilon_{\lambda}
$$

However, for a spherically symmetric atmosphere, the fraction of photons not intercepted by the star is given by

$$
G(r)=0.5\left[1+\left(1-\left(R_{*} / r\right)^{2}\right)^{1 / 2}\right]
$$


In interpreting transition region fiuxes one usually assumes a plane parallel atmosphere with $r=R_{*}$, so $\mathrm{G}(\mathrm{r})=0.5$.

The apparent emission measure over height is then

$$
E m(h)_{a p p}=\left(F_{\oplus} d^{2}\right) /\left(\epsilon_{\lambda} R_{*}^{2}\right)=E m(V)_{a p p} / 4 \pi R_{*}^{2}
$$

However, if the true volume emission measure is

$$
\operatorname{Em}(V)=\int N_{e}^{2} 4 \pi r^{2} d r
$$

then what is observed is

$$
E m(V)_{a p p}=\int N_{e}^{2} 4 \pi r^{2} G(r) d r
$$

Thus the relation between the true emission measure over radial extent, $\operatorname{Em}(\mathbf{r})$, and the apparent volume emission measure is

$$
E m(r)=E m(V)_{a p p}\left(R_{*} / r\right)^{2} / 4 \pi R_{*}^{2} G(r)
$$

This spherically symmetric formulation has been used by Harper (1992) and Pan \& Jordan (1995).

The important point is that the apparent emission measure distribution will not have the same gradient (with temperature) as the true emission measure distribution, because of the additional geometrical factors.

\subsection{What Determines the Shape of the Emission Measure Distribution?}

Jordan et al. (1987) have shown that between $T_{e}=2 \times 10^{4} \mathrm{~K}$ and $10^{5} \mathrm{~K}$ the shape of the emission measure distribution is determined by the inverse of the radiative power loss function $P_{\text {rad }}\left(T_{e}\right)$.

Above about $T_{e}=2 \times 10^{5} \mathrm{~K}$, one can make two alternative simple approximations to show that the true emission measure distribution depends on $T_{\mathrm{e}}$ to a power that is close to $3 / 2$. Consider the energy balance

$$
d F_{M} / d r=-d F_{C} / d r-d F_{R} / d r
$$

where $F_{M}, F_{C}$ and $F_{R}$ are the non-thermal, conductive and radiative fluxes, respectively.

Now

$$
F_{C}=-\kappa T_{e}^{5 / 2} d T_{e} / d r
$$

Writing the emission measure over a fixed interval of $\Delta \log T_{e}=0.3$ as

$$
\operatorname{Em}(0.3)=P_{e}^{2}\left(d r / d T_{e}\right) / \sqrt{2} k^{2} T_{e}
$$

and substituting for $d r / d T_{e}$ in terms of $F_{C}$ gives

$$
\operatorname{Em}(0.3)=\kappa P_{e}^{2} T_{e}^{3 / 2} / \sqrt{2} k^{2} F_{C}
$$

When $F_{C}$ and $P_{e}$ are constant, one obtains the well-known result that $E m(r)$ scales as $T_{e}^{3 / 2}$.

Alternatively one can assume that over the first pressure-squared scale-height, from which the emission is mainly observed, conduction balances radiation so that in plane parallel geometry (see Pan \& Jordan 1995 for the spherically symmetric case).

$$
d F_{C} / d T_{e}=-d F_{R} / d T_{e}
$$

Using

$$
d F_{R} / d T_{e}=0.8 N_{e}^{2} P_{r a d}\left(T_{e}\right) d r / d T_{e}
$$




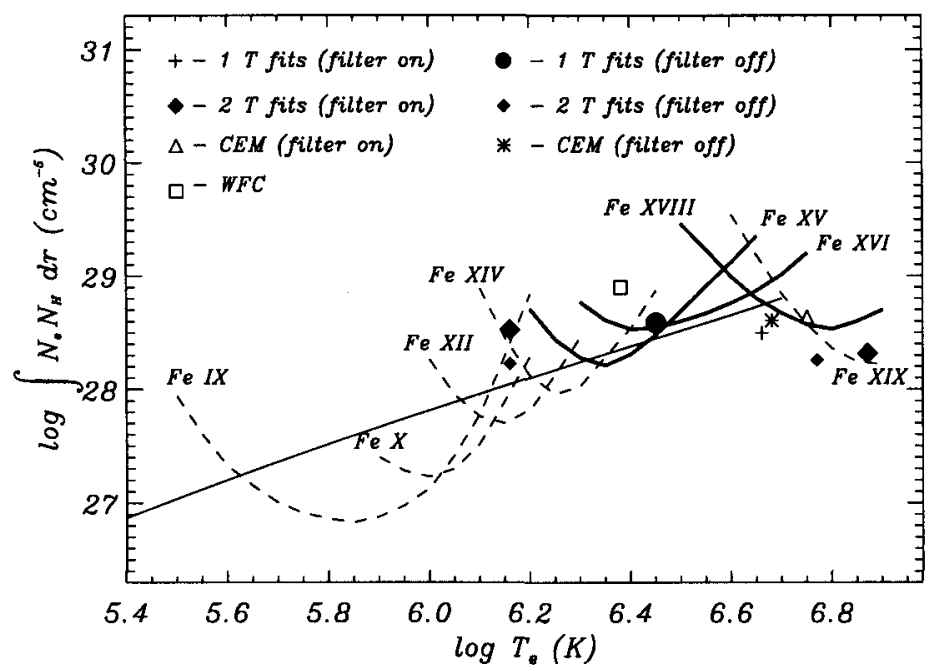

FiguRE 2. The apparent emission measure distribution for $\xi$ Boo A from $E U V E$ line fluxes. Short dashed lines indicate upper limits. The full line is the energy balance model using spherical symmetry. See key to symbols and text for results from ROSAT.

leads to

$$
d \log E m(0.3) / d \log T_{e}=3 / 2+2 d \log P_{e} / d \log T_{e}-1.6 k^{2} E m(0.3)^{2} P_{\text {rad }}\left(T_{e}\right) / \kappa P_{e}^{2} T_{e}^{3 / 2}
$$

The last term is essentially the coronal value of $F_{R} / F_{C}$, and substituting typical numbers shows that this ratio is usually much less than 1 .

The emission measure distribution can then be computed as a function of temperature using the apparent coronal emission measure and temperature as the initial conditions, where the initial pressure is found by assuming that the coronal emission is formed over a pressure-squared scale height. If only the gas pressure is included in hydrostatic equilibrium then the radial extent is not large even in coronal giants. But if a wavepressure term is included, consistent with the widths of UV lines (see Harper 1992), then the radial extent becomes quite large. The apparent emission measure distribution can then have a gradient steeper than $3 / 2$, while the true emission measure distribution remains close to a $3 / 2$ gradient. The same approach can be used with other geometries, e.g., those appropriate to loop structures, when the loop area is constrained by the fact that equation (5.14) has no solution if the starting emission measure is larger than a certain value.

\subsection{Example Results}

Figure 2 shows the apparent emission measure loci found from lines of Fe XV, XVI and XVIII observed in the spectra of $\xi$ Boo A obtained with EUVE. The results from alternative fits to our ROSAT PSPC spectra and the point obtained from the WFC all-sky survey (Pye et al. 1995) are also shown. The emission measure from IUE spectra (Jordan et al. 1987) is about $10^{27}$ at $10^{5} \mathrm{~K}$. The WFC filter ratio assigns all the emission measure 


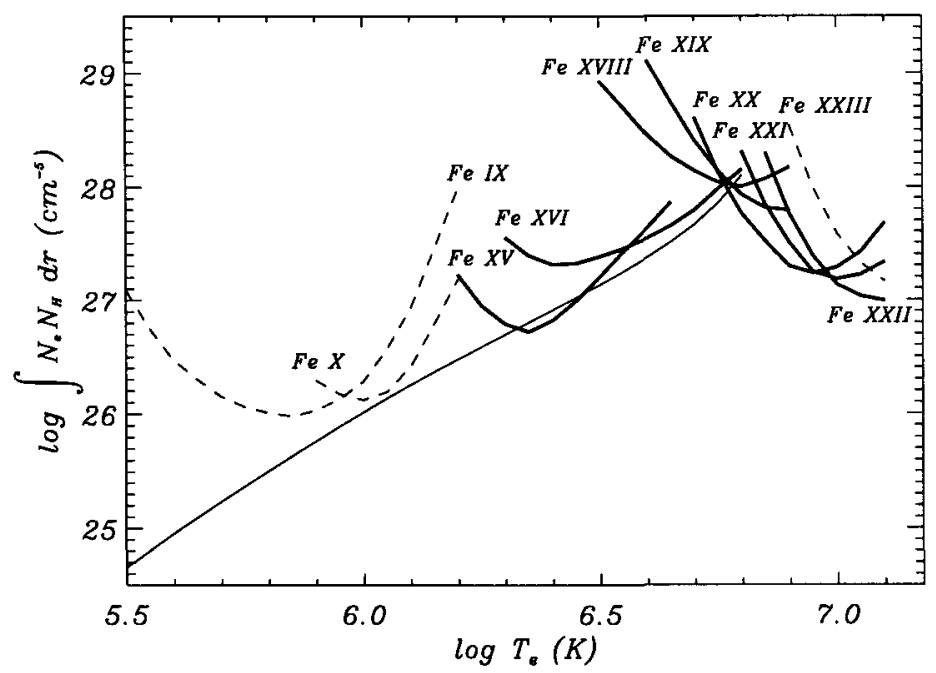

FIGURE 3. The apparent emission measure distribution for Capella from EUVE line fluxes. The full line is the result of an energy balance model using spherical symmetry and including wave pressure.

to the average temperature of the lines in the WFC wavebands and therefore tends to overestimate the emission measure. With two temperature fits the higher temperature tends to be larger than that of the peak coronal emission measure. The emission measure at the lower temperature is significantly higher than the upper limit found from the $E U V E$ line fluxes. A continuous emission measure fit gives the best agreement with the peak emission measure from the EUVE line fluxes. In the fits illustrated the 1994 version of the Raymond-Smith code has been used. For $\xi$ Boo A there is little difference between the solutions using plane parallel and spherically symmetric geometry. The difference between the observed distribution and that computed at temperatures between $3 \times 10^{5} \mathrm{~K}$ and $10^{6} \mathrm{~K}$ may be due to the area of the emitting region varying with temperature.

Figure 3 shows the apparent emission measure distribution for Capella, derived from the fluxes given by Dupree et al. (1993) and the emissivities of Brickhouse et al. (1995) at $N_{e}=10^{10} \mathrm{~cm}^{-3}$. Between $T_{e}=4 \times 10^{5} \mathrm{~K}$ and $6 \times 10^{6} \mathrm{~K}$ the apparent emission measure gradient is clearly steeper than $3 / 2$. This gradient can be reproduced by an energy balance model using spherical symmetry and including wave pressure in the equation of hydrostatic equilibrium. Without the wave pressure the predicted gradient is around 1.7. In either case the true emission measure distribution has a gradient close to $3 / 2$.

I am grateful to Dr Hongchao Pan for extracting the EUVE spectra used above, and to Miss D. Philippides for allowing me to use the spectral fits she has made to our spectra of $\xi$ Boo A obtained with ROSAT.

\section{REFERENCES}

Brickhouse, N. S., Raymond, J. C. \& Smith, B. W. 1995, ApJS, 97, 551 
Bromage, G. E., Cowan, R. D. \& Fawcett, B. C. 1978, MNRAS, 183, 19

DERE, K. P. 1978, ApJ, 221, 1062

Dupree, A. K., Brickhouse, N. S. Doschek, G. A., Green, J. C., \& Raymond, J. C. 1993, ApJL, 418, L41

Drake, J. J., Laming, J. M. \& Widing, K. G. 1995, ApJ, 443, 393

Drake, S. A., Singh, K. P., White, N. E. \& Simon, T. 1994, ApJL, 436, L87

Fawcett, B. C. 1974, Adv. At. Mol. Phys., 10, 223

FAwCETT, B. C. 1981, Phys. Scripta, 24, 663

FAwCETT, B. C. \& CowaN, R. D. 1975, MNRAS, 171, 1

Fawcett, B. C., Cowan, R. D., Kononov, E. Y. \& Hayes, R. W. 1972, J. Phys. B, 5, 1255

Fawcett, B. C., Jordan, C., Lemen, J. \& PhillPs, K. 1987, MNRAS, 225, 1013

Feldman, U., Doschek, G. A. \& Seely, J. F. 1988, J. Opt. Soc. Am. B, 5, 2237

Gabriel, A. H., Fawcett, B. C. \& Jordan, C. 1966, Proc. Phys. Soc., 87, 825

HARPER, G. M. 1992, MNRAS, 256, 37

Jordan, C. 1968, J. Phys. B, 1, 1004

Jordan, C. \& Brown, A. 1981, In Solar Phenomena in Stars and Stellar Systems, ed. R. M. Bonnet \& A. K. Dupree, Dordrecht: Reidel, 199.

Jordan, C., Brown, A., Walter, F. M. \& LinSky, J. L. 1986, MNRAS, 218, 465

Jordan, C., Ayres, T. R., Brown, A., Linsky, J. L. \& Simon, T. 1987, MNRAS, 225, 903

KAAstra, J. E. 1992, An X-ray Spectral Code for Optically Thin Plasmas, Internal SRON-

Leiden Report V2

Kastner, S. O., Neupert, W. M. \& Swartz, M. 1974, ApJ, 191, 261

Kelly, R. L. 1987, J. Phys. Chem. Ref. Data, 16, S, No. 1, Part II

LANG, J. 1994, Special Ed. Atomic Data Nucl. Data, 57

Loulergue, M. \& Nussbaumer, H. 1975, A\&A, 45, 125L

MalinovsKy, M. \& Heroux, L. 1973, ApJ, 181, 1009

Mason, H. E. \& Monsignori, Fossi, B. C. 1995, A\&AR, in press

Mason, H. E., DoscheK, G. A., Feldman, U. \& Bhatia, A. K. 1979, A\&A, 73, 74

Mewe, R., Gronenschild, E. H. B. M. \& VAN, DEN, OoRD, G. H. J. 1985, A\&AS, 62, 197

Monsignori, Fossi, B. C. \& LANDini, M. 1994, A\&A, 284, 900

Pan, H. C. \& Jordan, C. 1995, Mon. Not. R. astr. Soc., 272, 11

Pye, J. P. ET AL. 1995, MNRAS, in press

SAndlin, G. D., Brueckner, G. E., Scherrer, V. E. \& Tousey, R. 1976, ApJL, 205, L47

Schmitt, J. H. M. M., Harnden, F. R., Peres, G., Rosner, R. \& Serio, S. 1995, ApJ, 288,751

SchriJver, C. J. 1985, Space Sci. Rev., 40, 3

Stern, R. A., Lemen, J. R., Schmitt, J. H. M. M. \& Pye, J. P. 1995, ApJ, in press

TousEY, R. 1967, ApJ, 149, 239 